Canad. Math. Bull. Vol. 47 (1), 2004 pp. 73-81

\title{
Systems of Hermitian Quadratic Forms
}

\author{
Ma Li and Chen Dezhong
}

Abstract. In this paper, we give some conditions to judge when a system of Hermitian quadratic forms has a real linear combination which is positive definite or positive semi-definite. We also study some related geometric and topological properties of the moduli space.

\section{Introduction}

E. Calabi studied linear systems of real quadratic forms in [1], [2]. He considered the necessary and sufficient conditions for existing a positive definite linear combination of two real quadratic forms with real coefficients. Let's review his results in the following way. Given $1 \leq k \in \mathbb{N}$. Let $V$ be a $k$-dimensional vector space of $n$ by $n$ real symmetric matrices $\mathbb{S}^{n}$ (or equivalently quadratic forms), and we write

$$
V=\operatorname{span}_{R}\left\{S_{1}, \ldots, S_{k}\right\}
$$

Then it is clear that $k \leq m=n(n+1) / 2$. Then we come to a question: Does $V$ contain a positive definite matrix? Consider a mapping

$$
F: R^{n} \rightarrow R^{k} \quad(n \geq k)
$$

defined by

$$
F(x)=\left(x^{*} S_{1} x, \ldots, x^{*} S_{k} x\right)^{T}, \quad \forall x \in R^{n} .
$$

Assume that

$$
K:=\operatorname{ker} F=\left\{x \in R^{n} \mid F(x)=0\right\}=\{0\}
$$

Then "yes" trivially, if $k=1$; E. Calabi showed in [1] that the answer is "yes" again if $k=2$ and $n>2$; and "no" for all other cases, i.e., for $k=n=2$ and for $2<k<m$. So every case is settled!

So one may ask if the inverse is true or not. E. Calabi showed in [2] that:

Theorem $A$ (See Theorem, [2, p. 331]) When $k=3$, the image set under the map $F$ is a convex cone provided some linear combination of these three quadratic forms is positive definite.

Received by the editors April 30, 2002.

The work of Ma is partially supported by key 973 project of China, a grant from Ministry of Education, and a scientific grant of Tsinghua University at Beijing. We thank Profs. J. Hurtubise and N. Kamran at McGill University for helpful discussion.

AMS subject classification: 15A63.

Keywords: hermitian quadratic form, positive definite, positive semi-definite.

(C) Canadian Mathematical Society 2004. 
E. Calabi gave counter-examples in [2] to show that when $k=3$, there exists only a positive semi-definite linear combination of these three quadratic forms, and the image of $F$ is not convex. When $k=4$, no such theorem is true at all.

All of these results of E. Calabi are fundamental and interesting. It seems to many people that E. Calabi's results are the last word. To get new results, one has to add restrictions. Based on his work we consider similar problems of linear systems of Hermitian quadratic forms. But Hermitian quadratic forms can be seen as real quadratic forms in some cases. In fact, let $H$ be a Hermitian matrix. Write $H=U+i V$, with $U, V \in M_{n}(R)$. From $\bar{H}^{T}=H$, we have

$$
U^{T}-i V^{T}=U+i V \Rightarrow U^{T}=U, \quad V^{T}=-V .
$$

For $\forall z \in C^{n}$, write $z=x+i y$, with $x, y \in R^{n}$. Then

$$
H(z)=\bar{z}^{T} H z=\left(x^{T}-i y^{T}\right)(U+i V)(x+i y)=x^{T} U x+y^{T} U y-2 x^{T} V y .
$$

So we can view $H$ as a real quadratic form over $R^{2 n}$ :

$$
\tilde{H}=\left(\begin{array}{cc}
U & -V \\
V & U
\end{array}\right)
$$

Then, by Theorem 1 in [1], we have:

Theorem $B$ Let $P(z), Q(z)$ be two Hermitian quadratic forms over $C^{n}$, where $z \in C^{n}$ and $2 \leq n<\infty$. Then $P(z)$ and $Q(z)$ satisfy the property that the only $z \in C^{n}$ satisfying $P(z)=Q(z)=0$ is $z=0$, if and only if there is a real linear combination of $P$ and $Q$ that is a positive definite quadratic Hermitian form.

On the other hand, we have for each symmetric matrix $U$, we can identify it with a Hermitian matrix $H$ on $C^{n}$ by the definition:

$$
\tilde{H}=\left(\begin{array}{cc}
U & 0 \\
0 & U
\end{array}\right)
$$

However, we can obtain new results about Hermitian forms over $C^{n}$ by restricting the Hermitian forms with more properties. We believe that our results are useful. Our results, for example, Theorems $8,9,11$, and 12 etc., can be considered as generalizations of E. Calabi's results. So it is appropriate to treat the Hermitian case separately.

To express our results in more detail, let introduce some notations and assumptions.

Let $H_{1}, \ldots, H_{m}$ be linearly independent members of the real vector space $\mathbb{H}^{n}$ of complex $n$-by- $n$ Hermitian matrices. We shall refer to a Hermitian matrix $H$ as a form whenever it is convenient. The form we have in mind is $z \longrightarrow z^{*} H z=H(z)$. By comparing dimensions we see that $m$ cannot exceed $n^{2}$. Let GPD (resp. GPSD) denote the sentence: $G=\operatorname{span}\left\{H_{1}, \ldots, H_{m}\right\}$ contains a positive (resp. nonzero positive semi-) definite matrix. Let $K=K_{n}$ (resp. $\mathcal{R}=\mathcal{R}_{m}$ ) denote the kernel (resp. range) of the mapping $F(z)=F_{n, m}(z)=\left[H_{1}(z), \ldots, H_{m}(z)\right]^{T}$ from $C^{n}$ into 
$R^{m}$. If $m=n^{2}, I$ is in $G$ so GPD holds, but if $m<n^{2}$, GPD may be false e.g. if every $H_{i}$ lies in $\left\{H \in \mathbb{H}^{n}\right.$; trace $\left.H=0\right\}$. So the property GPD (resp. GPSD) is not easily tamed.

Some of our new results can be summarized as follows:

Typical Theorem Let $m \geq 3$.

(1) If $K=\{0\}$ and $\mathcal{R} \backslash\{0\}$ is convex, then GPD holds.

(2) If $K=\{0\}$ and $\left\{H_{i}\right\}_{i=1}^{m}$ are pairwise commutative, then $\mathcal{R}$ is convex, and GPD.

This result is an easy consequence of Theorem 8 and Theorem 12 below. The case when $m<3$ is treated in Theorem 1 below. One may find more results in the next section. In the last section we consider some geometric property of the moduli space, which will be defined below.

\section{Algebraic Results and Proofs for Hermitian forms}

We let $\langle$,$\rangle denote the inner product in R^{m}$. We state the following:

Theorem 1 If $m<3$, then GPD holds iff $K=\{0\}$.

Remark 2 As we mentioned in the introduction, this result follows from Calabi's result. Since we can give a simpler proof of Theorem 1, we present a full proof of it here.

Proof The necessary condition is obvious. We will only prove the sufficient condition. Case ' $m=1$ ' is trivial. We will only prove case ' $m=2$ '.

Define the map

$$
f: C^{n} \backslash\{0\} \rightarrow S^{1}, \quad z \mapsto\left(\frac{H_{1}(z)}{d_{2}(z)}, \frac{H_{2}(z)}{d_{2}(z)}\right),
$$

where

$$
d_{2}(z)=\sqrt{H_{1}(z)^{2}+H_{2}(z)^{2}} .
$$

Denote $D=f\left(C^{n} \backslash\{0\}\right)$. For $n<\infty, D$ is a connected, closed subset.

Claim D contains no antipodal points.

If not, say, $(a, b)$ and $(-a,-b)$ are both in $D$. Let

$$
X=\left\{z \in C^{n} \backslash\{0\} \mid b H_{1}(z)-a H_{2}(z)=0\right\} .
$$

Then by the definition of $f$, we have $f(X)=\{(a, b),(-a,-b)\}$, which is a disconnected subset of $D$. Since $X$ is connected, so is $f(X)$, a contradiction.

By the claim, $D$ is strictly contained in some open half-circle $\Gamma$. Take the midpoint $(c, d)$ of $\Gamma$. Geometrically, the angles between the point $(c, d)$ and the end points of $D$ are less than $\pi / 2$. Then we have $\langle(c, d), f(z)\rangle>0$, for all $z$ in $C^{n} \backslash\{0\}$. That is $c H_{1}+d H_{2}$ is positive definite. 
Corollary 3 If $m=3$, then GPD holds iff (i) $K=\{0\}$ and

$$
\left\{\frac{H_{2}(z)}{H_{3}(z)} \mid H_{1}(z)=0, H_{3}(z) \neq 0\right\} \neq R^{1} .
$$

Proof We begin to prove the necessary condition.

(i) is obvious. We only prove (ii) here.

Assume $a H_{1}+b H_{2}+c H_{3}$ is positive definite, with $a, b, c \in R^{1}$. Clearly, we can take $b \neq 0$. Otherwise, we can perturb $(a, b, c)$ to make $b \neq 0$. Then $a H_{1}+b\left(H_{2}+\frac{c}{b} H_{3}\right)$ is positive definite. By Theorem 1, we have

$$
H_{1}(z)=H_{2}(z)+\frac{c}{b} H_{3}(z)=0 \Leftrightarrow z=0 .
$$

If (ii) fails, then $\exists z_{0} \in C^{n}$, s.t. $\frac{H_{2}\left(z_{0}\right)}{H_{3}\left(z_{0}\right)}=-\frac{c}{b}, H_{1}\left(z_{0}\right)=0, H_{3}\left(z_{0}\right) \neq 0$. So we have $H_{2}\left(z_{0}\right)+\frac{c}{b} H_{3}\left(z_{0}\right)=0$. By (1), it must be that $z_{0}=0$. Therefore $H_{3}\left(z_{0}\right)=0$, a contradiction. (ii) is proved.

We now prove the sufficient condition.

Take

$$
s \in R^{1} \backslash\left\{\frac{H_{2}(z)}{H_{3}(z)} \mid H_{1}(z)=0, H_{3}(z) \neq 0\right\} .
$$

Claim $H_{1}(z)=H_{2}(z)-s H_{3}(z)=0 \Leftrightarrow z=0$.

In fact, if $\exists z_{1} \in C^{n}$, s.t. $H_{1}\left(z_{1}\right)=H_{2}\left(z_{1}\right)-s H_{3}\left(z_{1}\right)=0$, then

Case $1 \quad H_{3}\left(z_{1}\right)=0 \Rightarrow H_{2}\left(z_{1}\right)=0$. By $(\mathrm{i}), z_{1}=0$.

Case $2 H_{3}\left(z_{1}\right) \neq 0 \Rightarrow s=\frac{H_{2}\left(z_{1}\right)}{H_{3}\left(z_{1}\right)}$. This contradicts the choice of $s$.

So it must be that $z_{1}=0$.

The claim is proved.

By Theorem 1, there exists a positive definite real linear combination of $H_{1}$ and $\mathrm{H}_{2}-s \mathrm{H}_{3}$, i.e., GPD holds.

Remark 4 We give some geometric interpretation of Corollary 3.

(A) Assuming condition (i) of Corollary 3, we define a mapping

$$
f: C^{n} \backslash\{0\} \rightarrow S^{2}, \quad z \mapsto\left(\frac{H_{1}(z)}{d(z)}, \frac{H_{2}(z)}{d(z)}, \frac{H_{3}(z)}{d(z)}\right),
$$

where

$$
d(z)=\sqrt{H_{1}(z)^{2}+H_{2}(z)^{2}+H_{3}(z)^{2}} .
$$

Denote $D=f\left(C^{n} \backslash\{0\}\right)$. By condition (ii), $D$ contains no antipodal points. Then it is easy to see that $D$ is contained in some open half ball. Arguing as in the last part of the proof of Theorem 1, we know that the sufficient condition of Corollary 3 is true.

(B) By (A), we can easily find that $D$ is spherical convex. In fact, if not, there exists some great circle whose intersection with $D$ is not connected, which is impossible. Since we believe this fact is useful, we formulate it as a theorem. 
Theorem 5 If $m=3$, then $\mathrm{GPD} \Rightarrow \mathcal{R}$ is convex.

By considering Hermitian matrices as symmetric matrices in $R^{2 n}$, Theorem 5 can be obtained from the theorem of [2] directly. An interesting corollary is:

Corollary 6 If $m=2$, then $\mathcal{R}$ is convex and either (1) $\mathcal{R}=R^{2}$ or (2) GPSD holds.

Proof Let $H_{3}(z)=|z|^{2}$. Then $0 \cdot H_{1}+0 \cdot H_{2}+1 \cdot H_{3}$ is positive definite. By Theorem 5 ,

$$
\tilde{R}:=\left\{\left(H_{1}(z), H_{2}(z), H_{3}(z)\right) \mid z \in C^{n}\right\}
$$

is convex. Denote $\Pi: w=1$. Then $\tilde{R} \cap \Pi$ is convex, i.e.,

$$
\bar{R}:=\left\{\left.\left(H_{1}(z), H_{2}(z)\right)|| z\right|^{2}=1\right\}
$$

is convex. From this, one can get the conclusion easily.

Remark 7 By Remark 2 and the reason in [2], Theorem 5 cannot be improved in the following two cases:

(i) If $m=3$ and GPSD holds, then $\mathcal{R}$ may not be convex.

(ii) If $m=4$ and GPD holds, then $\mathcal{R}$ may not be convex.

The reverse of Theorem 5 is a special case of the following theorem.

Theorem 8 If $K=\{0\}$ and $\mathcal{R} \backslash\{0\}$ is convex, then GPD holds.

Proof $\mathcal{R} \backslash\{0\}$ has an interior point in the topology of $\mathcal{R}$, and $\{0\}$ is convex and disjoint from $\mathcal{R} \backslash\{0\}$, so there exists a functional $g$ in $W^{*}$ strictly separating $\{0\}$ from $\mathcal{R} \backslash\{0\}$ (cf. e.g. [4]), where $W$ is the smallest linear space containing $\mathcal{R}$. Extend $g$ to be 0 on the orthogonal complement of $W$. Then $g$ composed with $F$ is positive definite.

From the discussion above, we can see that $K=\{0\}$ can't prevent $\mathcal{R}$ from containing antipodal points, and then $R \backslash\{0\}$ may not be convex. A weaker conclusion is:

Theorem 9 If $\mathcal{R}$ is convex and not $R^{m}$, then GPSD holds. it.

The proof of Theorem 9 is similar to that of Theorem 8, but simpler. So we omit

We will give an application of Theorems 8 and 9. For that, we need the following lemma.

Lemma 10 Let $\left\{H_{i}\right\}(i \in S)$ be a family of $n$-by- $n$ Hermitian matrices, pairwise commutative. Then there exists a unitary matrix s.t. $\left\{H_{i}\right\}(i \in S)$ are simultaneously diagonalized. 
Note that the concept of 'pairwise commutative' is for matrices and for hermitian operators, not for forms. The proof of Lemma 11 can be found in books on linear algebra.

Combining Lemma 10 with Theorem 9, we have:

Theorem 11 If $\left\{H_{i}\right\}_{i=1}^{m}$ are pairwise commutative, then $\mathcal{R}$ is convex, and either

(1) $\mathcal{R}=R^{m}$ or

(2) GPSD holds.

Combining Lemma 10 with Theorem 8, we have:

Theorem 12 If $K=\{0\}$ and $\left\{H_{i}\right\}_{i=1}^{m}$ are pairwise commutative, then $\mathcal{R}$ is convex, and GPD holds.

The proofs of Theorem 11 and 12 is quite similar. We will only give the proof of Theorem 12.

Proof By Theorem 8, we only need to check that $\mathcal{R} \backslash\{0\}$ is convex.

By Lemma 10, there exists a unitary matrix $U=\left(u_{1}, \ldots, u_{n}\right)$ s.t. $\left\{H_{i}\right\}_{i=1}^{m}$ are simultaneously diagonalized. Then for all $z_{i}$ in $C^{n}$ (assume $z_{i}=a_{i}^{j} u_{j}, a_{i}^{j} \in C, i=$ $1,2 ; j=1, \ldots, n)$, and for all $t \in(0,1)$, we have

$$
t H_{l}\left(z_{1}\right)+(1-t) H_{l}\left(z_{2}\right)=H_{l}\left(z_{3}\right),
$$

where

$$
z_{3}=\sqrt{t\left|a_{1}^{j}\right|^{2}+(1-t)\left|a_{2}^{j}\right|^{2}} u_{j}
$$

This is an easy calculation. By the condition, we know if $z_{1}, z_{2} \neq 0$, then $z_{3} \neq 0$. Therefore $\exists l_{0} \in\{1, \ldots, m\}$, s.t. $H_{l_{0}}\left(z_{3}\right) \neq 0$. By this we have

$$
\left(H_{1}\left(z_{3}\right), \ldots, H_{m}\left(z_{3}\right)\right) \in \mathcal{R} \backslash\{0\} \text {. }
$$

Then Theorem 12 is proved.

Example 13 Here are two counter-examples for Theorem 9 and Theorem 11.

(1) For $n=4, m=2$, consider

$$
P_{1}(z)=\left|z_{1}\right|^{2}-\left|z_{3}\right|^{2}, \quad P_{2}(z)=\left|z_{2}\right|^{2}-\left|z_{4}\right|^{2} .
$$

$P_{1}, P_{2}$ are pairwise commutative. $\mathcal{R}=R^{2}$.

(2) For $n=3, m=2$, consider

$$
P_{1}(z)=\left|z_{1}\right|^{2}-\left|z_{2}\right|^{2}, \quad P_{2}(z)=\left|z_{3}\right|^{2} .
$$

$P_{1}, P_{2}$ are pairwise commutative. $\mathcal{R}=R_{+}^{2}=\left\{\left(x_{1}, x_{2}\right) \in R^{2} \mid x_{2} \geq 0\right\}$.

Remark 14 Most of our results and proofs can be extended to infinite-dimensional unitary space. However, because the unit sphere of infinite-dimensional unitary space is no longer compact, we can only get weaker results. We will not list the corresponding conclusions here. 


\section{Geometric Property of the Moduli Space}

To state the results, we introduce a definition.

Definition 15 Let $H_{1}(z):=P(z), H_{2}(z):=Q(z)$ be two hermitian quadratic forms over $C^{n}$. We say that $H_{1}(z)$ and $H_{2}(z)$ satisfy property $(C)$ if $H_{1}(z)=H_{2}(z)=0$ implies that $z=0$. We write $\Omega:=\Omega(n)$ as the space of the ordered pairs $\left(H_{1}, H_{2}\right)$ with property $(C)$ when $2 \leq n<\infty$. $\Omega$ is called moduli space.

Remarks (a) It is clear that if one of $H_{1}, H_{2}$ is positive definite, then property $(C)$ is automatically true.

(b) There are two group actions on the pair $(P, Q)$. One is the $\mathrm{GL}(n, C)$-action defined as follows. Given $A \in \mathrm{GL}(n, C)$. Let $T_{A} P=A^{*} P A$ be the conjugate action of $A$ to $P$. Then it is clear that if $P$ and $Q$ satisfy property $(C)$, then $T_{A} P$ and $T_{A} Q$ also satisfy property $(C)$. The other action is the $R_{*}^{2}:=R^{2}-0 \times R \cup R \times 0$ action defined by $(P, Q) \rightarrow(x P, y Q)$ where $(x, y) \in R_{*}^{2}$. The property $(C)$ is also $R_{*}^{2}$ action invariant.

(c) Assume $n<\infty$. Using the continuity property, we can easily see that for any pair $(P, Q)$ with property $(C)$ there is a positive constant $\epsilon_{0}$ such that for every pair $\left(P_{1}, Q_{1}\right)$ with $\left|P_{1}-P\right|+\left|Q_{1}-Q\right|<\epsilon_{0}$, we have that $P_{1}$ and $Q_{1}$ satisfy property $(C)$. Therefore, $\Omega(n)$ is an oriented smooth manifold of real dimension $2 n^{2}$ with group actions. Note that the manifold $\Omega(n)$ is non-compact with the induced topology from the standard norm on $C^{n}$. A interesting question may be that one studies some (symplectic) geometric properties of $\Omega(n)$ and its quotient by the group actions.

Now we want to study some related geometric and topological properties of the family.

(1) For all $\left(H_{1}, H_{2}\right)$ in $\Omega$, denote

$$
F_{\left(H_{1}, H_{2}\right)}=\left\{\left(H_{1}(z), H_{2}(z)\right) \mid z \in C^{n} \backslash\{0\}\right\} \cap S^{1} .
$$

Take the midpoint of $F_{\left(H_{1}, H_{2}\right)}$ along $S^{1}$, denoted by $\left(a_{\left(H_{1}, H_{2}\right)}, b_{\left(H_{1}, H_{2}\right)}\right)$. Let

$$
f: \Omega \rightarrow S^{1}, \quad\left(H_{1}, H_{2}\right) \mapsto\left(a_{\left(H_{1}, H_{2}\right)}, b_{\left(H_{1}, H_{2}\right)}\right)
$$

and

$$
g: S^{1} \rightarrow \Omega, \quad(a, b) \mapsto(a I, b I)
$$

Obviously, $f, g$ are continuous, and $g \circ f: \Omega \rightarrow \Omega$; $f \circ g: S^{1} \rightarrow S^{1}$.

We want to show that $g \circ f \simeq \operatorname{id}_{\Omega}$.

Let

$$
J\left(\left(H_{1}, H_{2}\right), t\right)=t\left(H_{1}, H_{2}\right)+(1-t)\left(a_{\left(H_{1}, H_{2}\right)} I+b_{\left(H_{1}, H_{2}\right)} I\right) .
$$

Claim For all t in $[0,1], J\left(\left(H_{1}, H_{2}\right), t\right)$ in $\Omega$. 
In fact,

$$
\begin{aligned}
a_{\left(H_{1}, H_{2}\right)} & {\left[t H_{1}+(1-t) a_{\left(H_{1}, H_{2}\right)} I\right]+b_{\left(H_{1}, H_{2}\right)}\left[t H_{2}+(1-t) b_{\left(H_{1}, H_{2}\right)} I\right] } \\
& =t\left[a_{\left(H_{1}, H_{2}\right)} H_{1}+b_{\left(H_{1}, H_{2}\right)} H_{2}\right]+(1-t)\left[a_{\left(H_{1}, H_{2}\right)}^{2}+b_{\left(H_{1}, H_{2}\right)}^{2}\right] I
\end{aligned}
$$

which is always positive definite. So the claim is proved.

Obviously, $J$ is continuous. We have that

$$
J\left(\left(H_{1}, H_{2}\right), 0\right)=g \circ f\left(\left(H_{1}, H_{2}\right)\right), \quad J\left(\left(H_{1}, H_{2}\right), 1\right)=\mathrm{id}_{\Omega} .
$$

Therefore

$$
g \circ f \simeq \operatorname{id}_{\Omega}
$$

Obviously,

$$
f \circ g=\mathrm{id}_{S^{1}} .
$$

Then $\Omega \simeq S^{1}$. Then we have

$$
\pi_{1}(\Omega)=\pi_{1}\left(S^{1}\right)=\mathbb{Z}
$$

(2) Obviously, $\Omega$ is an open subset of $\mathbb{H}^{n} \times \mathbb{H}^{n}$. For $(a, b) \in S^{1}$, define

$$
\Omega_{(a, b)}=\left\{\left(H_{1}, H_{2}\right) \in \Omega \mid a H_{1}+b H_{2}>0\right\} .
$$

it is easy to prove that $\Omega_{(a, b)}$ is an open convex subset of $\Omega$. Also it is easy to see that $\Omega_{(a, b)} \cong \Omega_{(c, d)}$, for all $(a, b),(c, d)$ in $S^{1}$.

Denote

$$
\begin{gathered}
A(\theta)=\left(\begin{array}{cc}
\cos \theta & \sin \theta \\
-\sin \theta & \cos \theta
\end{array}\right), \\
r_{0}=(1,0)^{t}, a(\theta)=(\cos \theta, \sin \theta)^{t}, \quad \theta \in R^{1} .
\end{gathered}
$$

Then $r_{0}=A(\theta) a(\theta)$. So

$$
\Omega_{a(\theta)}=\Omega_{r_{0}} \cdot A(\theta)=\left\{\left(H_{1}, H_{2}\right) A(\theta) \mid\left(H_{1}, H_{2}\right) \in \Omega_{r_{0}}\right\} .
$$

Notice

$$
\left(H_{1}, H_{2}\right) \in \Omega_{r_{0}}
$$

if and only if $H_{1}$ is positive. We denote all $n$-by- $n$ positive definite Hermitian matrices by $H_{p}^{n}$. Then $\Omega_{r_{0}}=H_{p}^{n} \times \mathbb{H}^{n}$. We have a continuous surjection

$$
\begin{gathered}
h: \Omega_{r_{0}} \times R^{1} \rightarrow \Omega, \\
\left(\left(H_{1}, H_{2}\right), \theta\right) \mapsto\left(H_{1}, H_{2}\right) A(\theta) .
\end{gathered}
$$


Define an equivalence relation:

$$
\sim:\left(H_{1}, H_{2}\right) \sim\left(H_{3}, H_{4}\right) \Leftrightarrow \exists \theta \in R^{1}
$$

s.t.

$$
\left(H_{1}, H_{2}\right)=\left(H_{3}, H_{4}\right) A(\theta) .
$$

Then

$$
\Omega / \sim \cong \Omega_{r_{0}} / \sim
$$

Finally, we point out the following fact: $\left(H_{1}, H_{2}\right) \sim\left(H_{3}, H_{4}\right) \Rightarrow H_{1}, H_{2}, H_{3}, H_{4}$ can be diagonalized simultaneously. This comes from the following fact: if there exists a positive definite real linear combination of $H_{1}, H_{2}$, then $H_{1}, H_{2}$ can be diagonalized simultaneously.

\section{References}

[1] E. Calabi, Linear systems of real quadratic forms. Proc. Amer. Math. Soc. 15(1964), 844-846.

[2] Linear systems of real quadratic forms 2. Proc. Amer. Math. Soc. 84(1982), 331-334.

[3] Werner Greub, Lineare Algebra. Berlin, Springer-Verlag, 1958.

[4] J. L. Kelley and Isaac Namioka, Linear topological spaces. Princeton, N.J., Van Nostrand, 1963.

Department of Mathematical Sciences

Tsinghua University

Beijing 100084

China

e-mail: cdz02@mails.tsinghua.edu.cn

Department of Mathematics

The National University of Singapore

10 Kent Ridge Crescent

Singapore 119260

e-mail:nuslma@sina.com
Department of Mathematical Sciences

Tsinghua University

Beijing 100084

China 